\title{
EDP Distribuição experience in Power Quality permanent monitoring in Portugal
}

\author{
F. Gonçalves ${ }^{1}$, F. Bastião ${ }^{1}$, A. Lebre ${ }^{1}$, P. Veloso ${ }^{1}$ and N. Melo ${ }^{1}$ \\ ${ }^{1}$ Department of Quality of Service - Power Quality \\ EDP Distribuição (EDP Group) \\ Rua do Túnel - 3030 - 006 Coimbra (Portugal) \\ Phone number: +351 239002 520, e-mail: fabrice.goncalves@edp.pt, fernando.bastiao@edp.pt, \\ antoniojose.lebrecardoso@edp.pt, pedro.veloso@edp.pt, nuno.melo@edp.pt
}

\begin{abstract}
This paper aims to give an overview of EDP Distribuição experience on Permanent Monitoring of Power Quality in Portugal.

Over the last thirteen years, EDP Distribuição has been developing a Power Quality monitoring program in order to meet a variety of challenges faced by distribution system operators, concerning to Power Quality. Based on this context, in 2010, EDP Distribuição introduced a new modality of systematic Power Quality monitoring based on permanent monitoring of HV/MV substations.
\end{abstract}

This paper presents the state-of-art of this modality, including the regulatory and the EDP Distribuição requirements, and the framing in the EDP Distribuição PQ monitoring platform.

Finally, the advantages of permanent monitoring and its actual/future challenges, namely concerning the new regulatory requirements and recommendations, as well the use of all this data, are discussed.

\section{Key words}

Permanent Monitoring, Power Quality, Monitoring Devices, Monitoring Platform.

\section{Introduction}

EDP Distribuição (EDP D) is a company of EDP Group Energias de Portugal. As the main DSO in Portugal, EDP D has about 6,1 million of distribution network customers, supplied by the distribution grid operated by EDP D, composed approximately by $83500 \mathrm{~km}$ of High Voltage (HV) and Medium Voltage (MV) lines and cables, $411 \mathrm{HV} / \mathrm{MV}$ and MV/MV substations and $66000 \mathrm{MV} / \mathrm{LV}$ substations, with a total power capacity around 17000 MVA (figures referred to the end of 2013), being the size of the LV distribution grid around $141000 \mathrm{~km}$.

To ensure a high level of Quality of Service (QoS), EDP D has been developing systematically, since 2001, a program for Power Quality (PQ) monitoring, according to the standard NP EN 50160 and the national QoS Regulation code.

\section{Power Quality monitoring program evolution}

Since the start of EDP D's PQ monitoring program in 2001, beginning with some temporary campaigns of 7 days long, and given the natural evolution of monitoring devices and tools and subsequent learning over them by EDP D, the monitoring program has evolved, as shown in Figure 1.

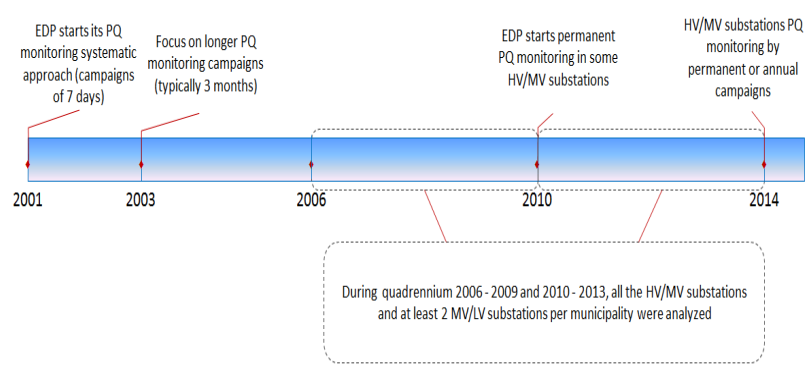

Fig. 1. Evolution of the PQ Monitoring Program - 13 years of systematic PQ monitoring

After 2003, the program came to be mainly based on 3 months PQ monitoring campaigns in $\mathrm{HV} / \mathrm{MV}$ and MV/LV substations. In 2010, EDP D introduced a strategy of PQ permanent monitoring in some HV/MV substations. This program includes, in addition, PQ monitoring at some sensitive customers.

Globally, this program allows to characterize the PQ in the distribution network and, as needed, at the customers' entrance, improves the operation and maintenance of the distribution network and finally support customers and report PQ to regulators and Internet.

\section{A. New regulatory requirements}

Since January 2014, a new QoS Regulation Code [1] is in application in Portugal, whose main requirements in terms of PQ monitoring ensure the start of monitoring at least $70 \mathrm{HV} / \mathrm{MV}$ substations in 2014, during at least 1 
year, and monitoring at least $2 \mathrm{MV} / \mathrm{LV}$ substations per municipality (total of 278 municipalities in Portugal mainland) during at least 3 months every 4 years.

About the HV/MV substations monitoring, QoS code also requires a minimum start of 40 substations in permanent monitoring and a yearly growing of at least $7 \mathrm{HV} / \mathrm{MV}$ substations in permanent monitoring.

In order to assure these requirements of the QoS code, currently, the EDP D program includes the PQ monitoring of MV busbars from HV/MV substations with permanent or annual campaigns and LV busbars from MV/LV substations with quarterly campaigns. During the year of 2014 were accompanied a total of $70 \mathrm{HV} / \mathrm{MV}$ substations and $168 \mathrm{MV} / \mathrm{LV}$ substations.

About the $70 \mathrm{HV} / \mathrm{MV}$ substations considered in 2014, 40 of them, approximately $60 \%$, were in permanent monitoring.

In the website of EDP D (www.edpdistribuicao.pt) it is possible to access to the EDP D' PQ Monitoring Plan established according the QoS Regulation Code to the biennium 2014-2015.

\section{Power Quality monitoring platform}

To support all this PQ activity, EDP D has been developing a technical PQ monitoring platform, comprising the PQ recorders (monitoring devices), communication infrastructures (collecting data), storage systems, as well as management and analysis software. The following figure shows a global topology of the PQ monitoring platform, which includes the PQ permanent monitoring component.

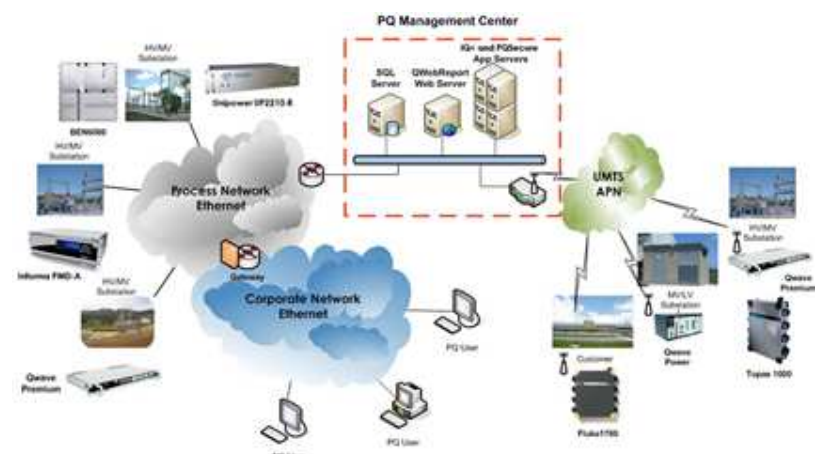

Fig. 2. PQ monitoring platform

Considering the temporary monitoring campaigns in substations, voltage measurements are performed in $\mathrm{MV}$ busbars of HV/MV substations and LV busbars of MV/LV substations, using portable PQ monitoring devices.

The strategy about the PQ monitoring devices used in the permanent monitoring in HV/MV substations is detailed in the next chapter of this paper.

Some customers are supported by PQ monitoring in order to perform an accurate characterization of the PQ supplied and to identify improvement actions. Examples of these customers are sensitive industries and LV microgenerators. Typically, a portable class A (IEC 61000-4-30) PQ monitoring device is installed for monitoring during a month.

Beyond the PQ monitoring devices, the platform is constituted by a central management system, which manages all remote communications with the devices and stores data automatically in a bulk SQL database.

The PQ data associated to continuous phenomena and voltage events is automatically collected daily from each PQ monitoring device. If necessary, this collection can be done manually by user's request.

With the purpose of providing fast and reliable communications, EDP D uses mostly Ethernet communications supported in fibre optic network. In the cases where this network is not available, 3G communications are used, supported in a closed Access Point Name (APN).

The PQ monitoring devices' management is carried out through its specific manufacturer software, which ensures not only the data collection and insertion in the database, but also allows their configuration, viewing and analysis.

The PQ data is processed in order to issue PQ overview reports, some of them specific to the Portuguese regulator. These reports are performed using a dedicated web based application. They depend on a weekly-based automatic process of data compilation, run by the application, that verifies firstly the consistence of data and secondly its conformity with NP EN 50160, enhancing a first outlook of PQ conditions of the distribution network, provided by each monitoring point.

Since 2014, the Portuguese regulation requires the publication of PQ results from monitoring campaigns activities undertaken at national level, including permanent monitoring and annual campaigns of HV/MV substations and the quarterly campaigns of $\mathrm{MV} / \mathrm{LV}$ substations. Those results are quarterly actualized.

Thus, the web based application has been designed to allow the production of reports for the substations being tracked, that are then loaded with a back-office application for later publication on the website of EDP D.

By accessing the specific PQ area of EDP D website, the user finds Portugal mainland map, shown in Figure 3, divided into territorial areas duly recognized for statistical purposes, which the user is able to select. 


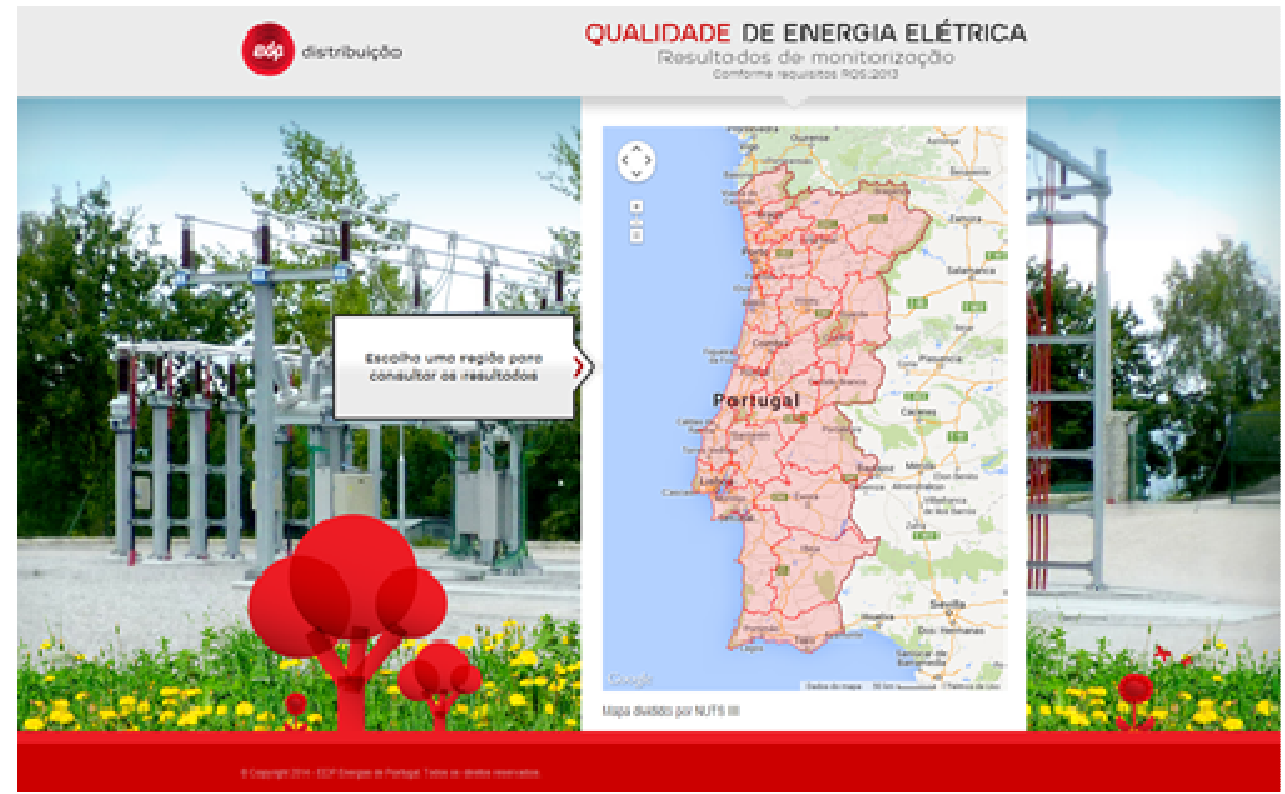

Fig. 3. EDP D website area for PQ results publication

After selecting the territorial area of interest, are presented the substations with PQ monitoring campaigns results, as shown in Figure 4.

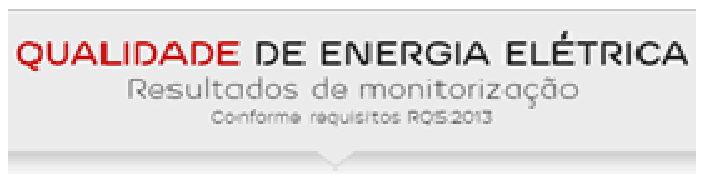

\section{PINHAL INTERIOR NORTE}

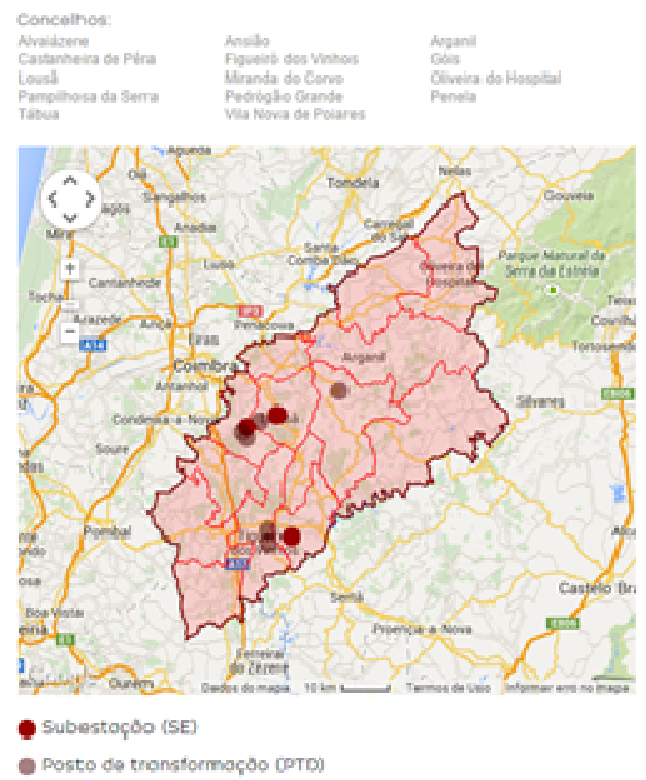

Fig. 4. Selected territorial area in detail

After selecting an existing substation, as shown in Figure 5 , the user has access to a report with information about continuous phenomena and voltage events recorded during the monitoring period, as shown in Figure 6.

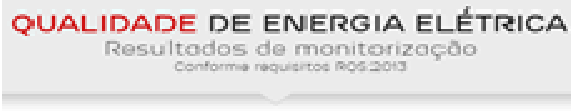

PINHAL INTERIOR NORTE

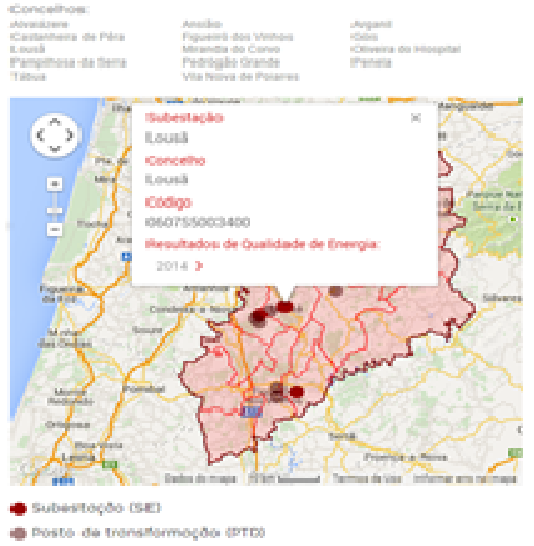

Fig. 5. Selection of HV/MV substation

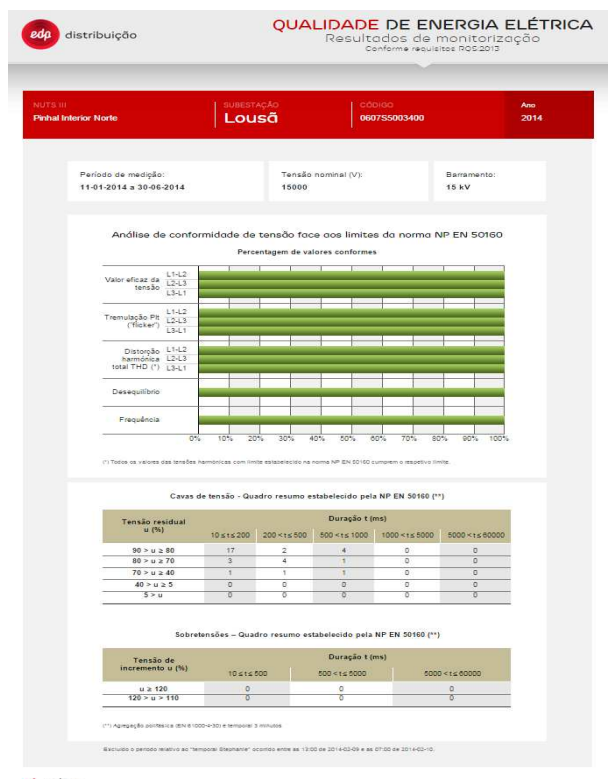

Fig. 6. PQ results for a HV/MV substation 


\section{Permanent monitoring of HV/MV substations}

As already mentioned, since 2010, EDP D began its implementation program of permanent monitoring in HV/MV substations. Since then, all new HV/MV substations and those subjected to a major refurbishment have been equipped with fixed PQ monitoring equipment. These devices are typically installed in all MV busbars, measuring voltages and also currents on the secondary side of power transformers. In addition to these analogic signals, some relevant digital signals are also collected, contributing to the analysis of voltage events and nonconformities in continuous phenomena.

Digital signals used can, per example, inform about the state of $\mathrm{HV}$ or MV circuit breakers or even if capacitor banks related to the MV busbar being monitored is connected or not.

Additionally, PQ monitoring devices allow different triggers configuration, in order to generate warnings and alarms, per example, if voltage and other continuous phenomena reach some boundaries, tighter than the specified by standards, allowing to anticipate nonconformity even before their occurrence.

PQ permanent monitoring of HV/MV substations with the creation of a longer data history and the knowledge of surrounding reality, related with the kind of sensitiveness of adjacent costumers, allows a deeper PQ analysis instead of simply meet regulatory limits.

In this way, it is expected that PQ permanent monitoring brings useful information relative to the real state of the distribution network and a better knowledge about PQ issues, namely to PQ sensitive costumers.

\section{A. Devices specification}

With the natural evolution of market products as well as their features and functionality, EDP D faced some difficulties when purchasing new PQ monitoring devices for permanent monitoring of $\mathrm{HV} / \mathrm{MV}$ substations, encountering the need to identify their characteristics necessary to ensure the requirements and growth of EDP D's PQ monitoring platform and especially to not compromise its integrability. Thus, in order to address these concerns as well as to meet regulatory needs, it was developed a specification [2] establishing the characteristics and tests that must comply with the PQ monitoring devices.

This specification has the following main objectives:

- To define the characteristics of the operation, design and construction, so that devices can be installed in the typical environment of $\mathrm{HV} / \mathrm{MV}$ substations.

- To define the functional characteristics, analog and digital inputs, requirements to remote communications and time synchronization.
- To define specific requirements in order to integrate devices into centralized management system, inclusively allowing the definition of scheduled upload of data and the management module to export data and in which format.

Thereby, all new recording devices, bought since 2010, are in accordance to the requirements of IEC 61000-4-30, class A.

The evolution of devices specification has tried to respond effectively to the constant mutations verified, both at regulatory and operational level. Thus, the latest edition, mainly, extend the range of possible suppliers, reaching two objectives:

- Not include DFR function, since it is also required for protection devices (IED).

- Obligation to export PQ data in a standard format (PQDIF format was elected), in order to not depend on proprietary formats to develop PQ reports.

\section{B. Some numbers}

Since the start of EDP D's permanent monitoring program, the number of substations equipped with fixed devices has been increasing progressively in accordance with the policy of expansion defined by the company.

More recently, the publication and entrance of the new QoS code in Portugal, has reinforced the necessity of this increase. In the next few years, EDP D considers that the number of HV/MV substations with permanent monitoring will follow the trend indicated in the Figure 7.

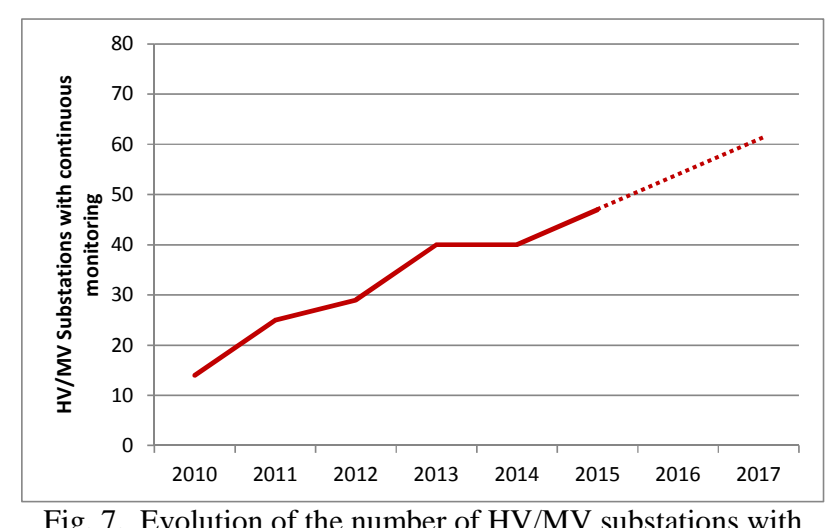

PQ permanent monitoring

In 2014, the number of HV/MV substations with PQ permanent monitoring corresponds to about $11 \%$ of total.

In Portugal, there is an activity licensed by special legal regulation, called Produção em Regime Especial (PRE) Special Regime Generation, which is the generation of electricity from renewable local resources or the combined generation of heat and power. The PRE is the current legal framework for wind, solar and small hydro plants, as well as for cogeneration plants, biomass and waste recovery (urban, agricultural and industrial). 


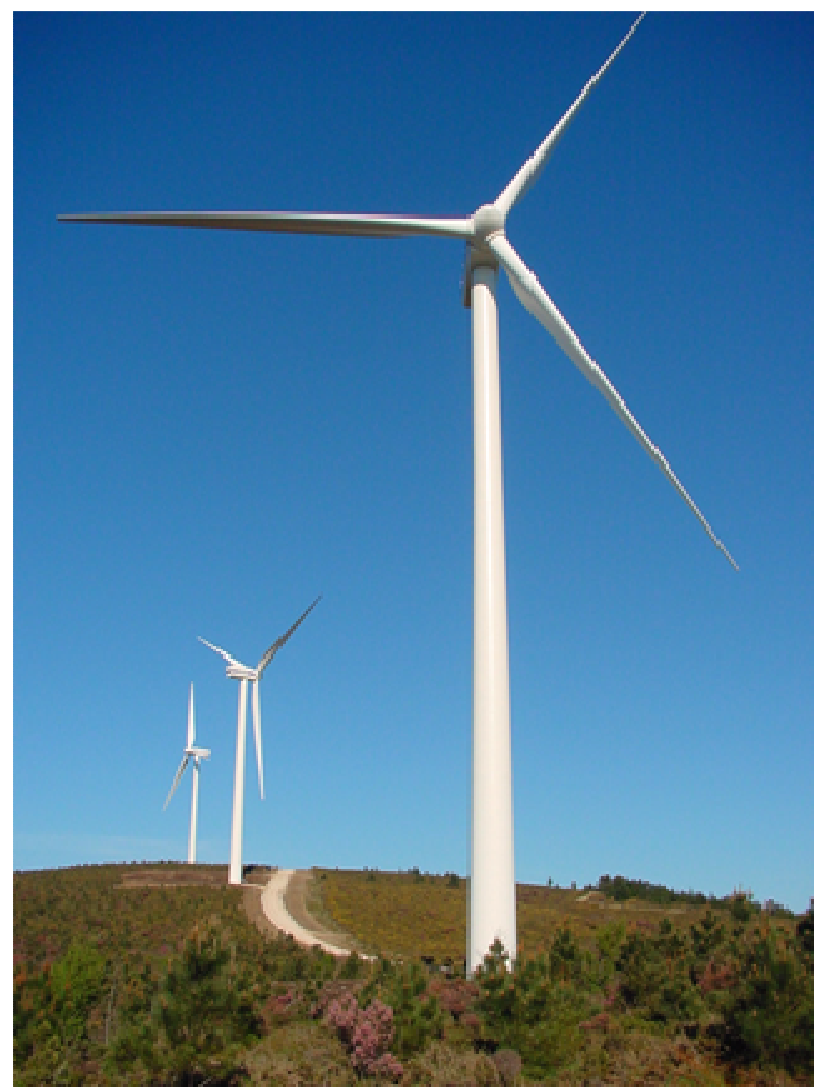

Fig. 8. EDP's Special Regime Generation example

The PRE is injected, in distribution network, in all voltage levels from LV to $\mathrm{HV}$. Given current regulatory requirements on the PQ permanent monitoring of $\mathrm{MV}$ busbars of HV/MV substations and the current EDP D' $\mathrm{s}$ policy regarding the choice of substations to contemplate with PQ permanent monitoring, the current substations in permanent monitoring allow to EDP D to track in PQ terms about $9 \%$ of the national PRE generation injected in MV.

Considering those HV/MV substations with PQ permanent monitoring, about 55\% of them has MV PRE generation associated downstream.

\section{Permanent monitoring advantages and main challenges}

From EDP D's experience, the main advantages of permanent monitoring are:

- Possibility of comprehensive historical allowing analysis with a fairly representative time base;

- Rapid identification and faster resolution of PQ non-conformity;

- Possibility of time synchronization with other network operation systems (SCADA, protections);

- Use of a wide range of digital signals providing the possibility of correlation of voltage events with circuit breakers or capacitor banks operation; - MV busbar current correlation with voltage events;

- Greater stability in PQ platform operation;
- The growth in demand for permanent monitoring solutions boosts market to refine their devices and functionalities, providing more robust and reliable resources for PQ monitoring to network operators;

- Unlike temporary monitoring, permanent monitoring allows greater harmonization of data between different network operators at European level and beyond, enabling the comparison of data and allowing streamline the study and mutual understanding on PQ issues.

Besides these mentioned advantages, EDP D considers that the development of the permanent monitoring program presents some challenges in a medium term, highlighting the following:

- Implement the succeeding changes of criteria in standards and regulation;

- Expansion the platform and consequent demands on memory management for data storage and effective maintenance of communications system;

- Implement the necessary updates in the platform in order to allow the use of data from different types of monitoring devices by the web based application, regardless of manufacturer, carrying out uniform analysis and respecting the same criteria independently of the origin of the data;

- Implementation of a real-time system of alerts regarding $\mathrm{PQ}$.

\section{Conclusion}

Since 2001, EDP D has developed a program for PQ monitoring, according to standard NP EN 50160 and the national QoS Regulation code, and, in 2010, introduced a strategy for PQ permanent monitoring in some HV/MV substations.

In order to assure requirements of the new QoS code, EDP D's program includes the PQ monitoring of MV busbars from $\mathrm{HV} / \mathrm{MV}$ substations with permanent or annual campaigns and LV busbars from MV/LV substations with quarterly campaigns. PQ results from these campaigns are published in EDP D website.

In 2014, the number of HV/MV substations with permanent monitoring corresponds to about $11 \%$ of total and facing the new regulation requirements will grow annually at least $7 \mathrm{HV} / \mathrm{MV}$ substations in permanent monitoring.

$55 \%$ of HV/MV substations with PQ permanent monitoring, in 2014, has MV Special Regime Generation associated downstream.

The requirements and challenges in terms of $P Q$ are increasing; thereby, EDP D, due its suitable PQ permanent monitoring experience, intends to further develop its integration on monitoring platform improving the QoS of the distribution network and being a reference in the Power Quality field. 


\section{Acknowledgement}

The authors thank the availability and collaboration of the colleagues Teresa Couceiro, Flávio Cação and António Margalho.

\section{References}

[1] Entidade Reguladora dos Serviços Energéticos, "Regulamento da Qualidade de Serviço do Setor Elétrico".

[2] EDP Distribuição, "DMA-C13-526/N - INSTALAÇÕES AT

E MT. SUBESTAÇÕES DE DISTRIBUIÇÃO Sistema de Monitorização da Qualidade de Energia Elétrica”. 